\title{
Alterações hematológicas e cardíacas em cavalos Árabes submetidos ao teste de esforço crescente em esteira rolante
}

Guilherme de Camargo FERRAZ ${ }^{1}$

Antônio Raphael TEIXEIRANETO $^{1}$

Flora Helena de Freitas

D'ANGELIS ${ }^{1}$

José Corrêa de LACERDA$\mathrm{NETO}^{2}$

Antonio de QUEIROZ-NETO'

Correspondência para:

Antonio de Queiroz - Neto, Departamento de Morfologia e Fisiologia Animal, FCAV/ UNESP, Via de Acesso Prof. Paulo D. Castellane s/n, 14884-900, Jaboticabal, SPaqueiroz@fcav.unesp.br

Recebido para publicação: 13/06/2007 Aprovado para publicação: 27/08/2009

\author{
1 - Departamento de Morfologia e Fisiologia Animal da Faculdade de \\ Ciências Agrárias e Veterinárias da Universidade Estadual Paulista, \\ Jaboticabal-São Paulo \\ 2 - Departamento de Clínica e Cirurgia Veterinária da Faculdade de Ciências \\ Agrárias e Veterinárias da Universidade Estadual Paulista, Jaboticabal-São \\ Paulo
}

\section{Resumo}

Estudaram-se alterações na freqüência cardíaca e variáveis hematológicas de eqüinos Puro Sangue Árabe (PSA), submetidos a exercício intenso em esteira rolante sob condições tropicais. Para tanto, 24 eqüinos, treinados, realizaram exercício teste de intensidade crescente e amostras de sangue foram obtidas durante as etapas de esforço 4; 5; 6; 7; 8; 9 e $10 \mathrm{~m} . \mathrm{s}^{-1}$, com $10 \%$ de inclinação. As colheitas de sangue foram realizadas 15 segundos antes do término de cada etapa. A freqüência cardíaca foi obtida em cada etapa do exercício teste. Com as amostras de sangue foram obtidos, hematócrito, hemácias, hemoglobina e leucócitos totais. Foram calculados índices hematimétricos absolutos, volume corpuscular médio (VCM), hemoglobina corpuscular média (HCM) e a concentração de hemoglobina corpuscular média (CHCM). Os resultados mostram que houve variação significativa ( $\mathrm{p} \leq 0,05)$, a partir do repouso, entre os valores médios da freqüência cardíaca e das variáveis hematimétricas. Destaque para o VCM e HCM que aumentaram significativamente $(\mathrm{p} \leq 0,05)$ na etapa de esforço máximo $(10 \mathrm{~m} / \mathrm{s})$ demonstrando haver anisocitose durante o exercício intenso. Desta forma, o grau das alterações destas variáveis fisiológicas está relacionado com a intensidade e duração do exercício teste.
Palavras-chave: Eqüino.

Freqüência cardíaca. Hematologia. Exercício. Esteira rolante.

\section{Introdução}

O estudo de variáveis fisiológicas, em testes controlados, constitui uma ferramenta importante para a determinação do potencial de cavalos atletas. Existem várias informações na literatura internacional a respeito do tema. ${ }^{1,2}$ Contudo, é reduzido o número de informações científicas nacionais obtidas sob condições tropicais.

A freqüência cardíaca (FC) é facilmente aferida durante o exercício e fornece um índice indireto da capacidade e função cardiovascular ${ }^{2}$, apresentando uma relação linear com o exercício de intensidade crescente. Por outro lado, em exercícios de baixa intensidade, esta relação pode ser influenciada por fatores ambientais que provocam ansiedade e excitação. ${ }^{2}$

Amostras de sangue são freqüentemente obtidas durante testes para avaliação do desempenho atlético. Variáveis hematimétricas como hematócrito, contagem de hemácias e concentração de hemoglobina plasmática podem ser utilizadas para avaliação dos efeitos, tanto do exercício como do treinamento. ${ }^{3}$ Adicionalmente, índices hematimétricos absolutos, como volume corpuscular médio (VCM), hemoglobina corpuscular média (HCM) e concentração de hemoglobina corpuscular média (CHCM) estão sendo utilizados para determinação da síndrome de excesso de treinamento (overtraining) em 
eqüinos. ${ }^{4}$ Estudo ${ }^{5}$ realizado a campo avaliou com eqüinos da raça Puro Sangue Inglês (PSI), submetidos a exercícios de diferentes intensidades, não revelou alteração significativa do VCM imediatamente após esforço físico moderado (trote) e intenso (galope). Contudo, existe pouca informação na literatura sobre o comportamento destes índices durante o exercício intenso praticado em esteira rolante. Desta maneira, o propósito deste estudo foi verificar as alterações na freqüência cardíaca e de variáveis hematológicas, que podem ser utilizadas para a avaliação do desempenho atlético de eqüinos, relacionadas ao exercício físico intenso em esteira rolante.

\section{Material e Método}

Utilizaram-se 24 eqüinos treinados, machos ou fêmeas, Puro Sangue Árabe (PSA), todos provenientes do Setor de Eqüinocultura da Faculdade de Ciências Agrárias e Veterinárias - Unesp, Jaboticabal, com peso corpóreo médio ( \pm E.P.M.) de $390 \pm 25,4 \mathrm{~kg}$ e idade média ( \pm E.P.M.) de $8,6 \pm 3,3$ anos. Os animais foram submetidos a exame clínico completo e, estando aparentemente hígidos, foram selecionados para comporem os grupos experimentais. Nos dias de exercício a temperatura e umidade relativa do ar média foram de $24,0^{\circ} \mathrm{C}$ e $60,6 \%$, respectivamente.

Os eqüinos foram adaptados ao exercício em esteira rolante de alto desempenho (Esteira Galloper ${ }^{\circledR}$, Sahinco LTDA, Palmital, São Paulo, Brasil) e submetidos ao exercício teste (ET) com duração de 30 minutos. Para tanto, empregou-se exercício de aquecimento durante 4 minutos à velocidade de $4,0 \mathrm{~m} \cdot \mathrm{s}^{-1}$, a qual foi incrementada, a intervalos de 1 minuto, para $5 ; 6 ; 7 ; 8 ; 9$ e $10 \mathrm{~m} \cdot \mathrm{s}^{-1}$. A partir desta etapa de esforço máximo, procedeuse à desaceleração, retomando a velocidade para 3,0 m.s ${ }^{-1}$, por 20 minutos, que correspondeu ao período de desaquecimento ativo. Toda a fase de esforço físico, com incremento da velocidade, foi realizada com a esteira a 10\% de inclinação.
A FC (bpm) foi estabelecida com um freqüencímetro digital (S610 Polar ${ }^{\circledR}$, Port Washington, New York, EUA), específico para eqüinos, sendo utilizados os momentos $0 ; 4 ; 5,6,7 ; 8,9$ e 10 minutos, na fase de exercício. $\mathrm{Na}$ fase de desaquecimento ativo, a FC foi aferida nos momentos 15; 20 e 30 minutos.

Para obtenção das amostras de sangue foi criado um Procedimento Operacional Padrão (POP) que estabeleceu procedimentos adequados para a colheita, processamento e armazenamento. As colheitas de sangue foram realizadas 15 segundos antes do término de cada etapa de velocidade do exercício teste. Previamente à realização do exercício, os animais foram tricotomizados para cateterização da veia jugular esquerda cuja região foi submetida, previamente, à assepsia. Acoplou-se ao cateter intravenoso (Cateter Insyte ${ }^{\mathrm{TM}}$ $14_{\mathrm{GA}} \mathrm{X}_{1.75} 2,1 \times 45 \mathrm{~mm}-330 \mathrm{~mL} / \mathrm{min}$., Becton Dickinson Indústrias Cirúrgicas, Brasil) um tubo extensor (10 Fr5 x $60 \mathrm{~cm}$, Becton Dickinson Indústrias Cirúrgicas, Brasil) para facilitar as colheitas com o animal em movimento. Após cada colheita, todo o conjunto era lavado com solução de heparina a $2,5 \%$. Segundo este procedimento, desprezavam-se 2,0 $\mathrm{mL}$ de sangue advindos do início de cada colheita.

Para a determinação das variáveis hematológicas: hematócrito, hemoglobina e contagens globais de hemácias e leucócitos foram utilizados os momentos de exercício 0; 4; 6; 8 e 10 min. e, na fase de desaquecimento ativo, empregaram-se os mesmos momentos descritos para freqüência cardíaca. $O$ processamento das amostras sangüíneas foi realizado com aparelhos diluidor e contador (CELM - Cia. Equipadora de Laboratórios Modernos Barueri-SP). O hematócrito foi obtido pelo método do microhematócrito ${ }^{6}$ e a concentração de hemoglobina foi determinada pela metodologia colorimétrica cianeto de hemoglobina (Kit Labtest ${ }^{\circledR}$, Lagoa Santa, MG, Brasil). Todas as amostras foram colhidas em tubos com pressão negativa (Tubos EDTA, 5,0 mL Vacutainer 
$\mathrm{BD}^{\hat{a}}, \mathrm{BD}$ - Brasil - São Paulo - SP) e as análises feitas em triplicata. A análise estatística foi realizada utilizando-se o programa computacional SAS System for Windows V8 e os resultados apresentados como média \pm erro padrão da média. Para a comparação das variáveis fisiológicas obtidas dentro de cada etapa de esforço, foi realizada análise de variância. Para as diferenças estatísticas detectadas no teste F, realizou-se a comparação dos valores médios obtidos em cada etapa de esforço físico, tanto na fase de exercício como no desaquecimento ativo, por meio do teste de Tukey sendo estabelecido como nível de significância $\mathrm{P} \leq 0,05$.

\section{Resultados e Discussão}

A FC mostrou aumento dependente do incremento da intensidade de esforço.
Conforme demonstrado na figura 1, a FC máxima foi atingida na etapa $10 \mathrm{~m} \cdot \mathrm{s}^{-1}$, sendo o valor médio obtido igual a $219 \pm 2 \mathrm{bpm}$. Este foi significativamente maior $(\mathrm{p} \leq 0,05)$ quando comparado ao das outras etapas de esforço, excetuando a etapa 9 min. que se apresentou, do ponto de vista estatístico, igual à etapa de $10 \mathrm{~m} \cdot \mathrm{s}^{-1}$, indicando o início da fase de platô. $\mathrm{Na}$ fase de desaquecimento ativo, houve redução significativa da FC a qual se igualou, estatisticamente, com a etapa de aquecimento. A homeostase cardiovascular durante o exercício é mantida por mecanismos neuroendócrinos que asseguram o aumento da demanda do fluxo sangüíneo para a musculatura esquelética. ${ }^{7}$ Em concordância com Mckeever e Hinchcliff ${ }^{8}$, no repouso a FC média de eqüinos varia entre 30 a $40 \mathrm{bpm}$. Neste estudo, a FC no repouso foi elevada, como revelado na figura 1 , sendo que os valores

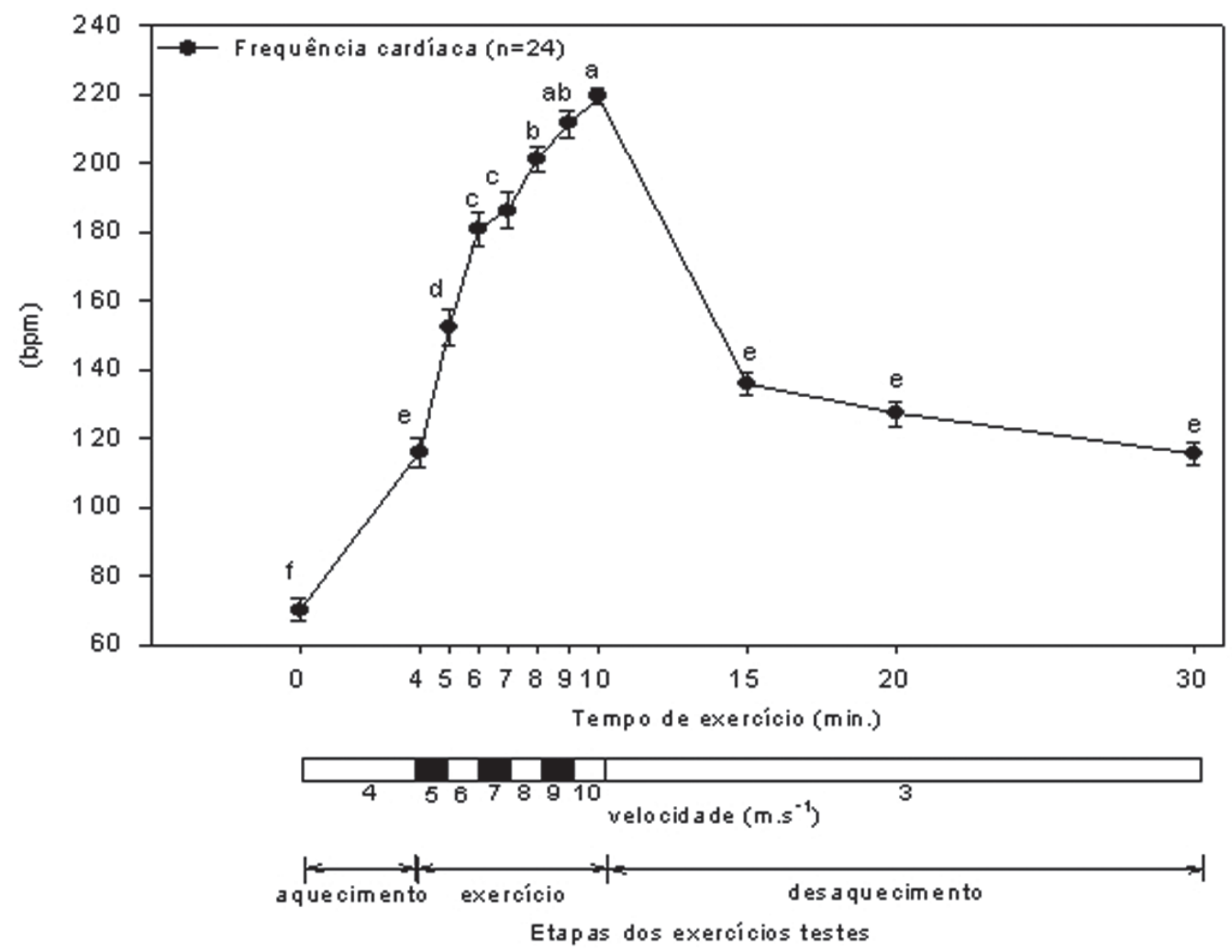

Figura 1 - Freqüência cardíaca (média \pm erro padrão da média) de eqüinos da raça Puro Sangue Árabe(PSA) submetidos à exercício de intensidade crescente em esteira rolante. Letras diferente diferem pelo teste Tukey $(p \leq 0,05)$ 
médios da FC antes do esforço físico foram de $70 \pm 3 \mathrm{bpm}$. Tanto para espécie humana como para os eqüinos, a expectativa do exercício inibe o controle cardiovascular pelo sistema nervoso parassimpático, prevalecendo o sistema nervoso simpático e resultando em aumentos da FC, força de contração, volume ejetado e débito cardíaco. ${ }^{9}$

Ainda observando a figura 1 , a FC aumentou proporcionalmente ao incremento do esforço físico, atingindo a máxima de $219 \pm 2 \mathrm{bpm}$. Este comportamento fisiológico é decorrente do aumento da atividade simpática, com liberação de catecolaminas, que promove, segundo Wilmore e Costill ${ }^{7}$, elevação do volume sistólico (0,7L para 2,0 L), a qual resulta em aumento do débito cardíaco de, em média, $30 \mathrm{~L} / \mathrm{min}$. em repouso, para quase 300L/min.

A análise da tabela 1 indica que, de uma maneira geral, houve aumento significativo ( $\mathrm{p} \leq 0,05)$, a partir do repouso, entre as médias de hematócrito, hemoglobina e contagens globais de hemácias e leucócitos. As variáveis analisadas apresentaram aumento de concentração até a etapa de esforço máximo do exercício teste $\left(10 \mathrm{~m} \cdot \mathrm{s}^{-1}\right)$, sendo que os valores médios do hematócrito e da concentração de hemoglobina foram de $53 \pm 0,7 \%, 17 \pm$
0,41 mg.dl ${ }^{-1}$ e 10,66 $\pm 0,24, \times 10^{6} / \mathrm{uL}$, respectivamente, ao passo que o número de hemácias foi máximo na etapa de $8 \mathrm{~ms}^{-1}$. As respostas das variáveis hematológicas ao esforço de intensidade crescente em esteira rolante foram similares àquelas encontradas em outros estudos ${ }^{3,10,11}$, que avaliaram cavalos da raça Puro Sangue Inglês (PSI) submetidos a testes físicos com incrementos de velocidade. Todas as variáveis hematológicas revelaram aumento nos seus valores médios (Tabela 1) relacionado com o aumento gradual do esforço. Este fato é normalmente imputado à contração esplênica esforço-dependente que é proporcional ao exercício e especialmente importante nos eqüinos. ${ }^{10,11}$ Segundo Persson ${ }^{12}$, nos eqüinos, a mobilização da reserva esplênica é ativada pelo estímulo simpático que sensibiliza receptores adrenérgicos $\alpha_{1}$, contraindo o baço e aumentando o número de hemácias circulantes e a hemoglobinemia. Este efeito é obviamente benéfico, pois eleva a capacidade aeróbia devido ao aumento do transporte de oxigênio para o músculo esquelético. ${ }^{5}$

Outro ponto interessante a ser considerado, principalmente a respeito da elevação do hematócrito, é a comparação entre raças. A maioria dos estudos ${ }^{10,11,12,13}$ enfoca o PSI relatando valores de hematócrito máximo entre $60-65 \%$. A raça utilizada neste estudo foi a PSA que também apresentou elevação do hematócrito, porém numa intensidade menor.

Tabela 1 - Variáveis hematológicas (Média \pm E.P.M.) de 24 eqüinos da raça Puro Sangue Árabe (PSA), submetidos à exercício com intensidade crescente em esteira rolante. FCAV/UNESP - Jaboticabal - 2006

\begin{tabular}{|c|c|c|c|c|c|c|c|c|}
\hline \multirow{3}{*}{$\begin{array}{c}\text { Variáveis } \\
(n=24)\end{array}$} & \multicolumn{8}{|c|}{ Tempo (min.) } \\
\hline & \multicolumn{5}{|c|}{ Exercicio } & \multicolumn{3}{|c|}{ Desa quecimento ativo } \\
\hline & $R$ & 4 & 6 & 8 & 10 & 15 & 20 & 30 \\
\hline Hematócrito (\%) & $39 \pm 1^{E}$ & $45 \pm 0,4^{D C}$ & $50 \pm 0,5^{B}$ & $50 \pm 0,4^{B}$ & $53 \pm 0,7^{\mathrm{A}}$ & $50 \pm 0,6^{B}$ & $47 \pm 0,7^{\mathrm{CB}}$ & $44 \pm 0,7^{D}$ \\
\hline Hemoglobina $(\mathrm{mg} / \mathrm{dl})$ & $13 \pm 0,44^{D}$ & $14 \pm 0,51^{D C}$ & $15 \pm 0,46^{\mathrm{ABC}}$ & $16 \pm 0,57^{\mathrm{A}}$ & $17 \pm 0,41^{\mathrm{A}}$ & $16 \pm 0,57^{\mathrm{AB}}$ & $16 \pm 0,39 A B C$ & $15 \pm 0,32^{\mathrm{BCD}}$ \\
\hline Hemácias $\left(x 10^{6} / \mathrm{uL}\right)$ & $8,49 \pm 0,28^{c}$ & $9,72 \pm 0,23^{B}$ & $10,72 \pm 0,31^{A B}$ & $10,89 \pm 0,26^{\mathrm{A}}$ & $10,66 \pm 0,24^{A B}$ & $10,53 \pm 0,26^{A B}$ & $10,58 \pm 0,26^{A B}$ & $9,99 \pm 0,28^{A B}$ \\
\hline Leucócitos (x103/uL) & $8,73 \pm 0,36^{c}$ & $9,90 \pm 0,33^{8}$ & $10,10 \pm 0,41^{A B}$ & $10,19 \pm 0,43^{A B}$ & $10,62 \pm 0,53^{A B}$ & $11,28 \pm 0,52^{\mathrm{A}}$ & $10,77 \pm 0,46^{\mathrm{AB}}$ & $9,91 \pm 0,44^{B}$ \\
\hline $\operatorname{VCM}\left(u^{3}\right)$ & $46,67 \pm 1,47^{\mathrm{B}}$ & $47,38 \pm 0,96^{B}$ & $47,92 \pm 1,47^{B}$ & $48,17 \pm 0,76^{\mathrm{B}}$ & $50,87 \pm 1,33^{A}$ & $48,48 \pm 1,51^{\text {B }}$ & $45,59 \pm 1,16^{B}$ & $45,22 \pm 1,36^{\mathrm{B}}$ \\
\hline $\mathrm{HCM}(\mathrm{pg})$ & $14,95 \pm 0,57^{\mathrm{B}}$ & $14,74 \pm 0,52^{B}$ & $15,18 \pm 0,60^{\mathrm{B}}$ & $15,74 \pm 0,61^{B}$ & $16,37 \pm 0,62^{\mathrm{A}}$ & $15,73 \pm 0,57^{B}$ & $15,33 \pm 0,49^{B}$ & $15,47 \pm 0,57^{B}$ \\
\hline CHCM (\%) & $33,92 \pm 1,16$ & $31,28 \pm 1,14$ & $31,91 \pm 1,14$ & $32,41 \pm 1,18$ & $32,24 \pm 0,94$ & $34,05 \pm 1,18$ & $33,74 \pm 0,90$ & $34,28 \pm 0,83$ \\
\hline
\end{tabular}

Médias seguidas de letras diferentes, na mesma linha, diferem entre si $(\mathrm{p} \leq 0,05)$ pelo teste de Tukey. $\mathrm{R}=$ Repouso. E.P.M.= Erro padrão da média 
Esta diferença poderia ser imputada a maior quantidade de volume plasmático observado nesta raça. ${ }^{14,15}$

Uma aplicação prática das variáveis hematológicas para avaliação do desempenho atlético é o emprego do hematócrito máximo, que pode ser utilizado para avaliação de supertreinamento sendo que Golland et al. ${ }^{16}$ encontraram, durante o esforço, valores de hematócrito máximo menores para eqüinos submetidos a um programa excessivo de treinamento.

Ao observar os índices hematimétricos absolutos, nota-se que o VCM e HCM aumentaram ( $\mathrm{p} \leq 0,05)$ na etapa de esforço máximo indicando aumento tanto no tamanho, como na concentração de hemoglobina das hemácias. Este resultado difere de um estudo realizado em pista de corrida ${ }^{5}$, que avaliou tanto o VCM como a amplitude de distribuição do diâmetro das hemácias, designada RDW (Red Blood Cell Distribuition Width), sendo observado somente aumento significativo desta última. Possivelmente, esta discrepância entre resultados ocorreu devido à maior intensidade de esforço do presente experimento (velocidade), interferindo principalmente na elevação do hematócrito. Além disso, os testes realizados em esteira rolante permitem a obtenção de amostras sangüíneas durante o esforço físico, o que não ocorre naqueles realizados a campo.,17

Os resultados deste estudo, com cavalos submetidos a exercício físico intenso em esteira rolante, podem sugerir aumento do tamanho das hemácias liberadas pelo baço, o que está de acordo com outros estudos realizados na espécie eqüina. ${ }^{5,18}$

Com relação aos leucócitos, quando comparados ao repouso, apresentaram concentração máxima no primeiro ponto de coleta da fase de desaquecimento ativo, sendo que os valores médios encontrados foram de $8,73 \pm 0,36 \times 10^{3} / \mathrm{uL}$ e $11,28 \pm$ $0,52 \times 10^{3} /$ uL, respectivamente. Esta elevação das células brancas, durante o exercício teste, pode ser considerada fisiológica, pois resultam da ação de hormônios como as catecolaminas que são liberadas em situações de medo, excitação ou durante o exercício vigoroso $^{19}$, e que atuam principalmente no baço. Entretanto, este efeito é transitório com duração máxima de 30 minutos $^{20}$.

\section{Conclusões}

Este estudo demonstrou que os sistemas cardiovascular e hematológico do equino integram-se de maneira coordenada durante exercício em esteira, possivelmente, pela ação neural autonômica simpática o que resultou em elevação da freqüência cardíaca e de variáveis hematológicas, com destaque para o VCM e HCM . Desta forma, o grau das alterações destas variáveis fisiológicas está relacionado com a intensidade e duração do exercício teste.

\section{Agradecimentos}

À FAPESP - Fundação de Amparo à Pesquisa do Estado de São Paulo, pelo auxílio concedido.

\section{Hematological and cardiac alterations in Arabian horses submitted to incremental effort test in treadmill}

\footnotetext{
Abstract

Alterations in heart rate and hematological variables of Arabian horses submitted to intense exercise in treadmill under tropical conditions were studied. Twenty four trained horses were adapted to exercise on a high performance treadmill and then submitted to an incremental (4; 5; 6; 7; 8; 9 and $10 \mathrm{~m} . \mathrm{s}^{-1}$ ) exercise test. Heart rate was verified and blood samples were taken 15 seconds before the end of each exercise
}

Key words: Equine. Heart rate. Hematology. Exercise. Treadmill. 
step to determine the hematocrit, concentration of leucocytes, erythrocytes and hemoglobin. From these data the mean corpuscular volume (MCV), the mean corpuscular hemoglobin $(\mathrm{MCH})$ and the mean corpuscular hemoglobin concentration (MCHC) were calculated. The results show that there were significant variations in heart rate and hematimetric variables from the rest when compared to the values after the exercise. The hematological indexes MCV and MCH had also increased significantly in the stage of maximum effort $(10 \mathrm{~m} / \mathrm{s})$ demonstrating the occurrence of anisocytosis during the intense exercise. In such a way, the degree of the alterations of these physiological variables is related with the intensity and duration of the effort.

\section{Referências}

1 HODGSON, D. R.; ROSE, R. J. The athletic horse. Philadelphia: W.B. Saunders, 1994. 497 p.

2 TRILK, J. L.; LINDNER, A. J.; GREENE, H. M.; ALBERGHINA, D.; WICKLER, S. J. A lactate-guided conditioning programme to improve endurance performance. Equine Veterinary Journal, v. 34, p. 122125, 2002. Supplement.

3 TYLER-MCGOWAN, C. M.; GOLLAND, L. C.; EVANS, D. L.; HODGSON, D. R.; ROSE, R. J. Haematological and biochemical responses to training and overtraining. Equine Veterinary Journal. v. 30, p. 621-625, 1999. Supplement.

4 PADALINO, B.; RUBINO, G.; CENTODUCATI, P. E.; PETAZZI, F. Training versus overtraining: evaluation of two protocols. Journal of Equine Veterinary Science, v. 27, n. 1, p. 28-31, 2007.

5 BALARIN, M. R. S.; LOPES, R. S.; KOHAYAGAWA, A.; LAPOSY, C. B.; FONTEQUE, J. H. Valores da amplitude de distribuição do tamanho dos eritrócitos (RDW) em eqüinos Puro Sangue Inglês (PSI) submetidos a exercícios de diferentes intensidades. Brazilian Journal Veterinary Research Animal Science, v. 43, n. 5, p. 637-641, 2006.

6 GOLDENFARB, P. B.; BOWYER, F. P.; HALL E.; BROSIOUS, E. Reproducibility in the hematology laboratory: the microhematocrit determination. American Journal of Clinical Pathology, v. 56, n. 1, p. 35-39, 1971.

7 WILMORE, J. H.; COSTILL, D. L. Hormonal regulation of exercise. In: WILMORE, J. H.; COSTILL, D. L. Physiology of sport and exercise. Champaign: Human Kinetics, 1994. p. 122-143.

8 MCKEEVER, K. H.; HINCHCLIFF, K. W Neuroendocrine control of blood volume, blood pressure, and cardiovascular function in horses. Equine Veterinary Journal, v. 14, n. 18, p. 77-81, 1995. Supplement.

9 MCKEEVER, K. H. The endocrine system and the challenge of exercise. Veterinary Clinics of North America Equine Practice, v. 18, n. 2, p. 321-353, 2002.
10 KOWAL, R. J.; ALMOSNY, N. R. P.; SUMMA, R. P.; CURY, L. J. Avaliação dos valores hematológicos em cavalos (Equus caballus) da raça Puro-Sangue-Inglês (PSI) submetidos a teste de esforço em esteira ergométrica. Revista Brasileira de Ciência Veterinária, v. 13, n. 1 , p. 25-31, 2006.

11 ROSE, R. J.; ALLEN, J. R.; HODGSON, D. R.; STEWART, J. H.; CHAN, W. Responses to submaximal treadmill exercise and training in the horse: changes in haematology, arterial blood gas and acid base measurements, plasma biochemical values and heart rate. Veterinary Record, v. 113, n. 26-27, p. 612-618, 1983.

12 PERSSON, S. On blood volume and working capacity in horses. Studies of methodology and physiological and pathological variations. Acta Veterinaria Scandinavica, v. 19, n. 9, p. 189, 1967. Supplement.

13 KINGSTON K. J. Hematologic and serum biochemical responses to exercise and training $\mathrm{In}$ : HINCHCLIFF, K. W.; GEOR, R. J.; KANEPS, A. J. (Ed.). Equine exercise physiology - The science of exercise in the athletic horse. London: Saunders, 2008. p. 398409.

14 CARLSON, G. P.; OCEN, P. O. Composition of equine sweat following exercise in high environmental temperatures and in response to intravenous epinephrine administration. Journal Equine Medicine Surgery, v. 3, n. 1, p. 27-32, 1979.

15 TEIXEIRA NETO, A. R.; FERRAZ, G. C.; MATAQUEIRO, M. I.; LACERDA NETO, J. C. QUEIROZ NETO, A. Reposição eletrolítica sobre variáveis fisiológicas de cavalos em provas de enduro de 30 e 60 km. Ciência Rural, v. 34, n. 5, p. 1505$1511,2004$.

16 GOLLAND, L. C.; EVANS, D. L.; MCGOWAN, C. M.; HODGSON, D. R.; ROSE, R. J. The effects of overtraining on blood volumes in standardbred racehorses. The Veterinary Journal, v. 165, n. 3, p. 228-233, 2003.

17 OLDRUITENBORGH-OOSTERBAAN, M. M. S. V.; CLAYTON, H. M. Advantages and disadvantages of track vs. treadmill tests. Equine Veterinary Journal, v. 30, p. 645-647, 1999. Supplement. 
18 SMITH, J. E.; ERICSON, H. H.; DEBOWES, R. M. Changes in circulating equine erythrocytes induced by brief, high-speed exercise. Equine Veterinary Journal, v. 21 , n. 6 , p. $444-446,1989$

19 PALUDO, G. R.; MCMANUS, C.; MELO, R. Q.; CARDOSO, A. G.; SILVA MELLO, F. P.; MOREIRA, M.; FUCK, B. H. Efeito do estresse térmico e do exercício sobre parâmetros fisiológicos de cavalos do exército brasileiro. Revista Brasileira de Zootecnia, v. 31, n. 3, p. 1130-1142, 2002.

20 LASSEN, D. E.; SWARDSON, C. J. Hematology and hemostasis in the horse: normal functions and common abnormalities. The Veterinary Clinics of North America Equine Practice, v. 11, n. 3, p. 351-389, 1995. 